\title{
A Proposal for a Novel Surface-Stress Based BioMEMS Sensor Using an Optical Sensing System for Highly Sensitive Diagnoses of Bio-particles
}

\author{
Mahdieh Khorsandifard $^{1} \cdot$ Kian Jafari $^{1}$ (D) $\cdot$ Arash Sheikhaleh $^{1}$
}

Received: 13 April 2020 / Revised: 8 May 2021 / Accepted: 27 June 2021 /

Published online: 27 July 2021

(c) The Author(s), under exclusive licence to Springer Science+Business Media, LLC, part of Springer Nature 2021

\begin{abstract}
In this paper, a BioMEMS sensor by using a surface-stress sensing approach, connected to a highly sensitive optical sensing system, is proposed to diagnose various types of biomolecules. The MEMS transducer is composed of a fixed-fixed beam with immobilized receptors on the surface which is connected to a Ring Resonator (RR) filter. The interaction between the target biomolecules and the receptors induces surface stresses on the beam. This stress results in the beam deformation which leads to changes in the coupling coefficient of the RR. Consequently, the transmission spectrum of the RR experiences changes, measured by using an optical photo-detector. Therefore, by analyzing the response of the photo-detector output, one can detect the number of target biomolecules in the sample and assign a level of contamination, infection or bioparticles, caused by the specific disease. Furthermore, the MEMS functional characteristics and the optical properties of the proposed biosensor are designed and analyzed respectively by using the finite element method (FEM) and the finite difference time domain (FDTD) approach. The obtained functional characteristics of the proposed device show that the present optical BioMEMS sensor can be used for highly sensitive diagnoses of various types of diseases and their progress level.
\end{abstract}

Keywords BioMEMS sensor $\cdot$ Ring resonator $\cdot$ Surface stress $\cdot$ Optical sensing

Kian Jafari

k_jafari@sbu.ac.ir

1 Faculty of Electrical Engineering, Shahid Beheshti University (SBU), Tehran, Iran 


\section{Introduction}

Nowadays, micro or nano-electro-mechanical system (M/NEMS) technologies are playing an important role in the development of high performance devices and tools for many industries. Thanks to their numerous advantages such as small dimensions, low price, and ease of batch fabrication, M/NEMS devices can be used in a wide range of applications such as inertial navigation, industrial production, remote detection, and so on [1-4]. One of the most interesting applications of the MEMS technology is biomedical diagnostics which is concentrated on the detection and characterization of various specific kinds of bioparticles including biomolecules, cells, ions, DNAs, and many others [5, 6]. This branch of the science is called BioMEMS that is basically aimed at taking the advantage of the MEMS technology to achieve high performance medical tools and devices such as biosensors [7].

In several diseases, the precise detection of biological analytes is vital in order to provide an early stage diagnosis and to prevent their fatal influences on the body. At clinical levels, these label-free biosensors are used to detect pathogens or various biomarkers (i.e. nucleic acids, proteins, and cells) of different diseases in the body fluids, such as saliva, blood, or urine [8]. For instance, a DNA-based piezoelectric biosensor can be used for simultaneous detection and genotyping of high-risk human papilloma virus (HPV) strains [9]. In addition, several viral diseases caused by various viruses such as Ebola, human immunodeficiency virus (HIV), and severe acute respiratory syndrome coronavirus 2 (SARS-CoV-2) has been studied by these types of biosensing platforms [10]. The label-free BioMEMS sensors can be thus a good candidate for real-time and cost-effective diagnosis of various diseases biomarkers.

The operating principle of BioMEMS sensors is usually based on the direct conversion of physical or chemical characteristics of bioparticles into a measurable mechanical displacement. The part which is responsible for this task is called MEMS transducer with a movable mechanical part. This MEMS part may be a cantilever beam [11], a fixed-fixed beam [12] or a diaphragm [13]. By detecting the magnitude and direction of this induced displacement, one can figure out the type and the number of target bioparticles. Surface stress variation is also one of the most appealing approaches which is used in the mentioned MEMS transducers. In this method, a spatial deformation induced by surface stress, resulting from the interaction between the MEMS transducer and the specific target bioparticles, is measured to determine the amount of the target bioparticles.

Many techniques have been reported in the literature so far to measure the resulting mechanical displacement such as capacitive technologies [14-16], piezoresistive technique [17], piezoelectric and optical approaches [18-20]. However, the optical approach can be the most promising one, thanks to several advantages such as large sensitivity, more immunity against electromagnetic interference (EMI), better thermal stability and thus better performance, compared to other existing sensing methods. For instance, Takahashi and his co-workers introduced a surface stress based BioMEMS sensor relied on an interferometric optical transducer [21]. The proposed device presents a new method in order to enhance the sensitivity of the mechanical part. However, the fabrication process complexity of the interferometric based 
optical sensing system is the main drawback of such devices. In another similar work, a polymer based optical MEMS sensor is numerically investigated by Serene et.al, which can be very suitable for biosensing applications [22]. In this device, a polymeric material is used, instead of silicon, to realize a micro-cantilever transducer, in order to decrease the device fabrication cost and improve its bio-compatibility. As the device uses an amplitude based optical sensing system, it is too sensitive to undesired fluctuations of the optical source. This can decrease the sensitivity and resolution of the biosensor. There are also several other optical sensing methods used in the biosensing applications, such as plasmonic refractive index sensors [23]. Although these types of all-optical biosensors can provide high resolution and appropriate thermal stability, their fabrication process is complicated and thus expensive compared to the biosensors based on the MEMS Technology. Furthermore, since the changes in the refractive index due to the adsorption of the biomolecules is very low, their sensitivity can be smaller than MEMS based sensors in which a mechanical transducer is used to convert the number of the absorbed target molecules into a measurable quantity [23-25].

In this paper, we propose a novel topology as a BioMEMS sensor based on the surface-stress detection and an optical sensing system. The proposed device can overcome several issues mentioned above (in terms of sensitivity, thermal stability, immunity against EMI). It can be also fabricated without the need for complicated fabrication processes that can decrease the total device cost. Furthermore, the proposed optical BioMEMS is designed and analyzed by numerical and analytical approaches and its functional characteristics are finally reported. The obtained functional characteristics make the proposed BioMEMS device appealing for biomedical diagnostics. The arrangement of the paper is as follows. In Sect. 2, the operating principles of the MEMS and the optical parts of the biosensor are separately introduced. Furthermore, in this section, design and analysis of the MEMS transducer and the optical sensing system of the proposed biosensor are carried out, respectively, by finite element method (FEM) and finite difference time domain (FDTD) approaches. The functional characteristics of the designed device are also obtained in this section. In Sect. 3, the results of numerical and analytical simulations are presented with the related discussions. Summary and conclusions are finally presented in Sect. 4.

\section{Design and Analysis of the Proposed BioMEMS Sensor}

In this section, the proposed biosensor is designed and analyzed in two separate, MEMS and optical, parts. To achieve this goal, the working mechanisms and the operation principles of the MEMS and the optical parts of the proposed biosensor are firstly studied in each sub-section. The BioMEMS sensor is then designed in order to obtain the MEMS characteristics and its optical properties based on the working mechanisms. 
Fig. 1 The working mechanisms of the surface stress effect a Tensile surface stress, b Compressive surface stress

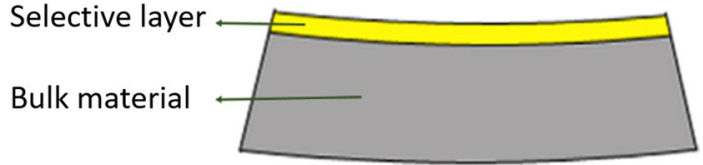

(a)

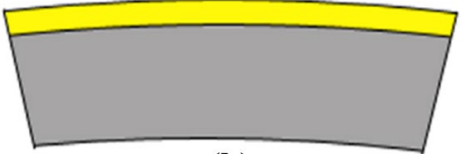

(b)

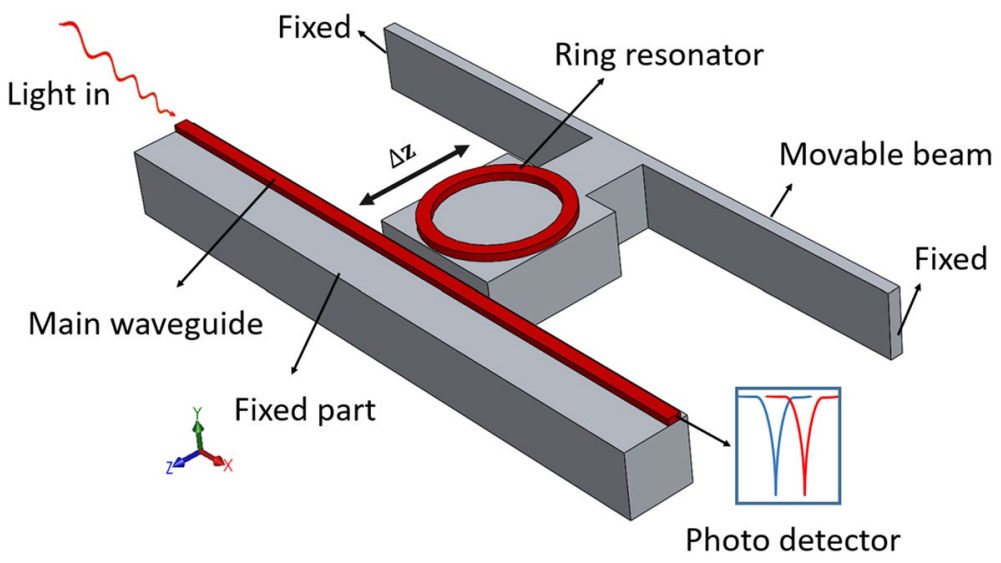

Fig. 2 The proposed surface stress based BioMEMS sensor

\subsection{Design and Analysis of the MEMS Transducer}

As mentioned before, the MEMS part of the proposed device is relied on the surface stress effect. The origin of the surface stress phenomenon is based on the nature of the chemical bonding of the atoms on the surface of a thin film. It means that a change in the density of the atoms on the surface of a thin film, caused by a chemical bonding process, can produce surface stresses. While surface atomic bonds are stiffer than their bulk counterparts, tensile surface stress is generated by the attractive forces between the surface atoms which results in concave surface curvature. On the contrary, when surface atomic bonds are softer than that of corresponding bulk, surface atoms repel each other and compressive surface stress is induced, which leads to convex surface curvature. This phenomenon is illustrated in Fig. 1.

The surface stress is formally modeled by the tensor components of the surface stress tensor $\sigma_{i j}$. Also, one can consider the Shuttle-worth equation defining the surface stress in terms of the surface energy, $\gamma[26]$ :

$$
\sigma_{i j}=\gamma \delta_{i j}+\partial \gamma / \partial \varepsilon_{i j}
$$


Table 1 Dimensions of the proposed biosensor
Fixed-fixed beam length

$100 \mu \mathrm{m}$

Fixed-fixed beam width

$2.4 \mu \mathrm{m}$

Height of the structure

where $\delta_{i j}$ is the Kronecker delta, and $\varepsilon_{i j}$ is the elastic strain tensor. In the proposed BioMEMS sensor, as shown in Fig. 2, a thin fixed-fixed SU8 beam is designed so that it is connected from the center to the ring resonator (RR). The dimensions of the beam are designed by FEM analysis (Table 1) in order to make an appropriate compromise between the sensitivity of the proposed biosensor and its bandwidth. Note that these dimensions can be redesigned based on the specifications of target biomolecules (i.e. target disease) to achieve the desired sensitivity and an appropriate bandwidth. However, it should be considered that there is always a compromise between the mechanical sensitivity of the proposed BioMEMS device and its bandwidth.
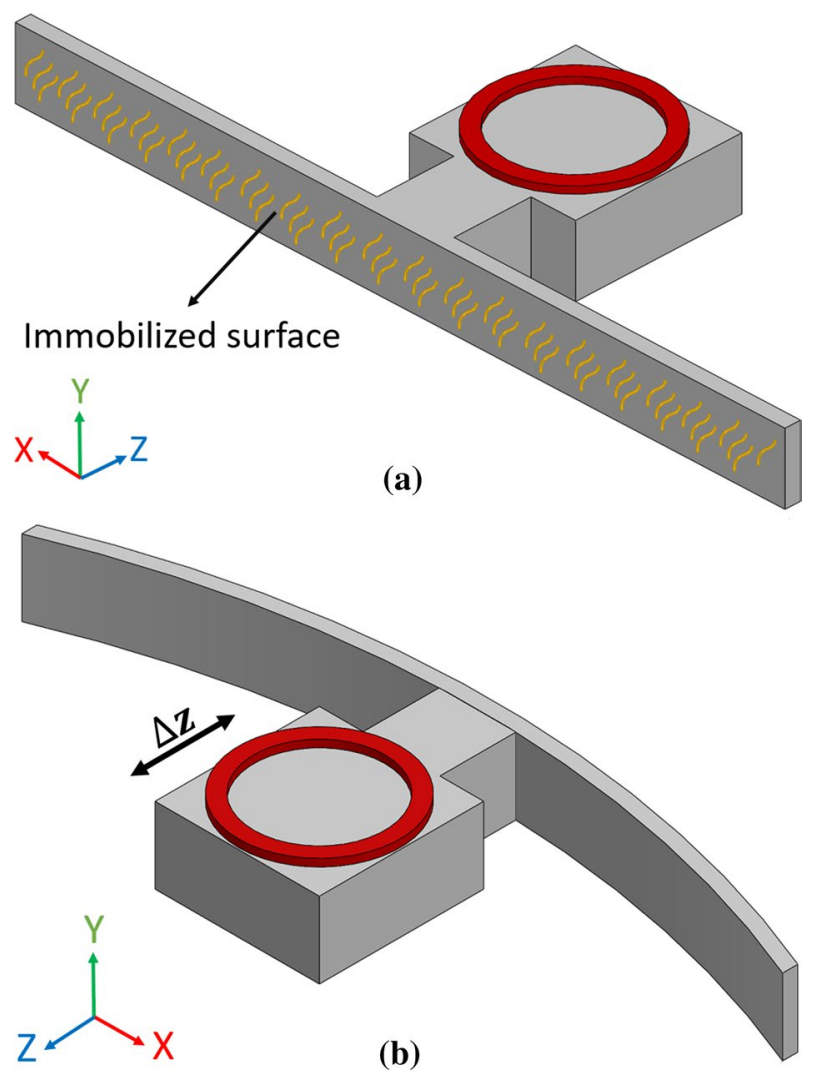

Fig. 3 a Schematic of the immobilized receptors on the surface of the proposed structure, $\mathbf{b}$ Deformation of the movable part under compressive surface stress 
In the proposed structure, a certain amount of the bioreceptor molecules should be immobilized on the surface of the beam in order to adsorb biomolecules as illustrated in Fig. 3a. By passing test samples through the free-standing surface, the target biomolecules existing in the test samples are adsorbed by the bioreceptors. This results in biochemical reactions on the surface of the beam that can trigger intermolecular forces which induces surface stresses and consequently a specific value of the deformation on the beam [27]. Then, the deformation leads to a small displacement of the ring (Fig. 3b) which is related to the fixed optical waveguide as shown in Fig. 2. This is how the MEMS transducer converts the existence of target biomolecules (and their existence level) into a mechanical displacement. Note that, one can choose the specification of the selective layer and its constituent bioreceptors (i.e. its types, the amount) based on target biomolecules and thus a specific disease related to the target biomolecules can be detected by the proposed BioMEMS sensor.

As presented earlier, the characteristics of the receptors and target molecules determine whether the beam experiences tensile or compressive stress, while target molecules pass through its surface. For instance, one can assume that adsorption of target molecules by the receptors can lead to compressive surface stress (Fig. 3b). In this scenario, the ring is displaced along $\mathrm{Z}$ axis, and the distance between the main waveguide and the ring is increased (and vice-versa for the tensile surface stress). This distance change which is a function of the characteristics and the amount of target molecules (i.e. a specific disease and its progress level) is then measured by the proposed optical sensing system as explained in the next section.

\subsection{Design and Analysis of the Optical Sensing System}

The optical sensing system of the proposed device is designed based on a tunable Ring Resonator (RR). The RR consists of a waveguide designed as a circular shape which is located nearby a straight waveguide. While a beam of light passes through the straight waveguide, a part of the light is coupled into the ring because of the evanescent field phenomenon. The coupled light in specific wavelengths, that satisfies the resonance circumstances, is propagated into the ring and is then stored and

Fig. 4 The RR structure and the transmittance spectrum of the main waveguide, while the ringwaveguide distance puts the RR in the critical coupling regime

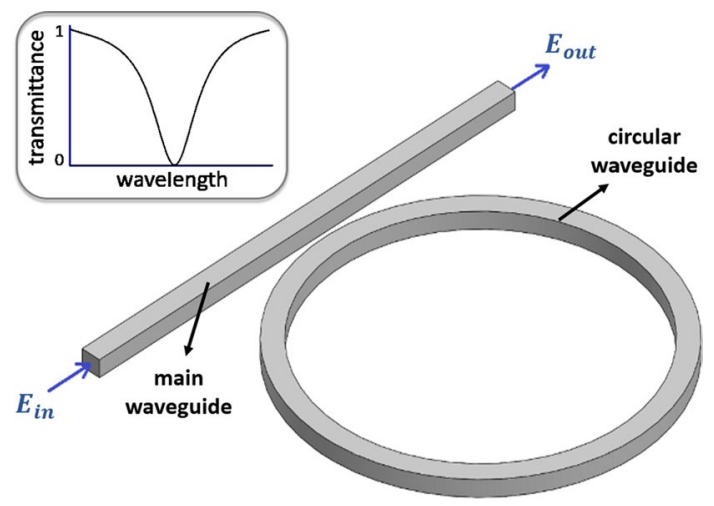


lost inside the resonator. Figure 4 shows the structure of a RR composed of the main waveguide, a circular waveguide, and a directional coupler.

In fact, the resonant phenomenon attenuates the guided modes in the ring and thus the transmission of the main waveguide at resonance drops to near zero at the resonance wavelength. In this situation, various periodic resonances troughs appear in the transmittance spectrum of the waveguide (Fig. 4). According to the coupling theory, the normalized optical transmission of the waveguide-coupled resonator is described by:

$$
T=\frac{\left|E_{\text {out }}\right|^{2}}{\left|E_{\text {in }}\right|^{2}}=\frac{\alpha_{0}^{2}-|t|^{2}-2 \alpha_{0}|t| \cos \emptyset_{0}}{1+\alpha_{0}|t|^{2}-2 \alpha_{0}|t| \cos \emptyset_{0}}
$$

where $E_{\text {in }}$ and $E_{\text {out }}$ are the amplitudes of the electric field of the input and the output light in the waveguide, $\alpha_{0}$ is the inner circulation factor, $\emptyset_{0}$ is the round trip phase, and $t$ is the transmission coefficient of the coupler that is related to the coupling coefficient $\kappa$ through $|\kappa|^{2}+|t|^{2}=1$.

The transmission of the resonance troughs (shown in Fig. 5) is determined by the transmission coefficient $t$ (or the coupling coefficient $\kappa$ ) of the coupler which is relied on three features (i.e. the distance and the coupling length between the waveguide, the ring and the effective refractive index). In the proposed optical sensing system, the effect of distance changes between the ring and the straight

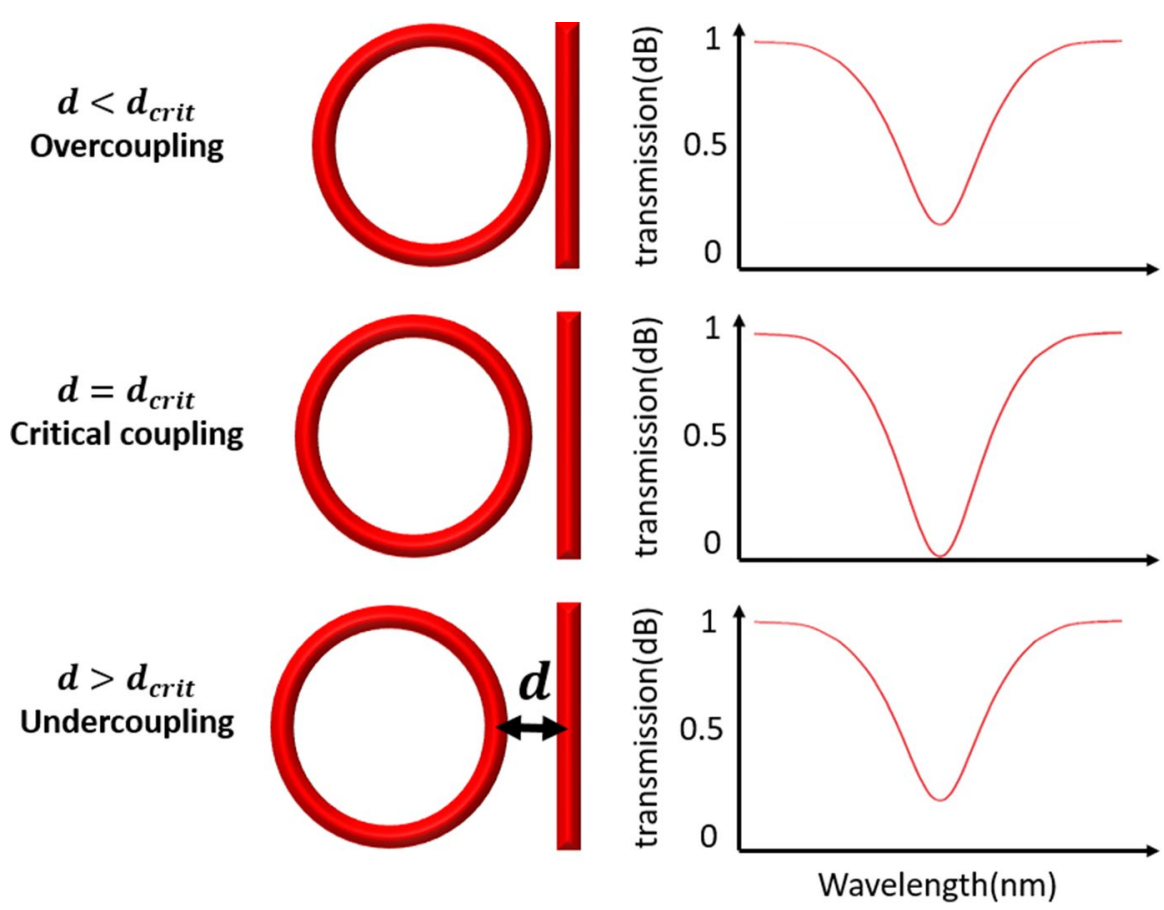

Fig. 5 Coupling regimes and respective transmission spectrum of the RR near the resonance 


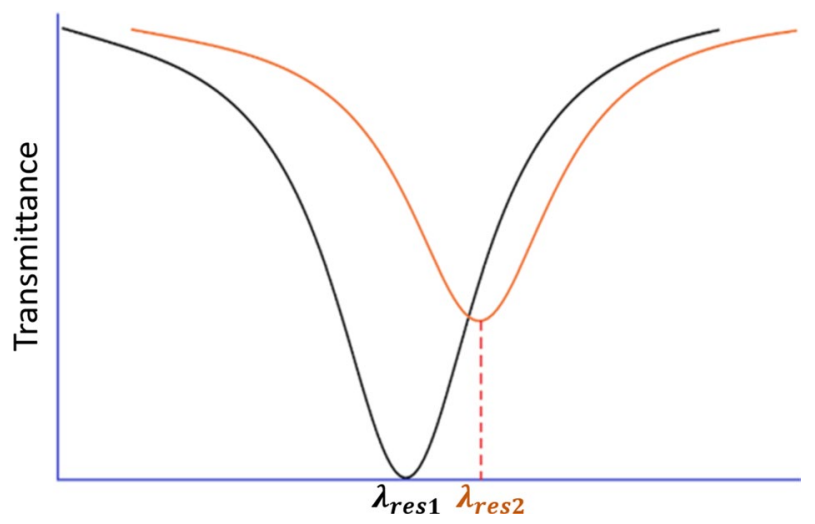

Fig. 6 Variations of the light transmittance spectrum in terms of the coupling distance change

Table 2 dimensions of the proposed sensor

\begin{tabular}{ll}
\hline Height of structure & $10 \mu \mathrm{m}$ \\
Inner radius of the ring waveguide & $4.9 \mu \mathrm{m}$ \\
Outer radius of the ring waveguide & $5.3 \mu \mathrm{m}$ \\
Thickness of the waveguides & $0.22 \mu \mathrm{m}$ \\
Primary gap distance of coupler & $0.35 \mu \mathrm{m}$ \\
Silicon refractive index & 3.4800 \\
SU8 refractive index & 1.5552 \\
\hline
\end{tabular}

waveguide is used in order to implement the concept of tunability. In order to obtain an appropriate tunability, the proposed structure should be designed so that it ensures the critical coupling in the initial state, while the ring is stationary. Therefore, the geometrical properties of the RR structure (such as geometrical dimensions of the ring and waveguide as well as the coupler gap) should be precisely chosen to achieve the mentioned goal. For a given value of the inner circulation factor, if the gap between the main waveguide and the ring is such that the transmission at the resonance wavelength drops to zero, the critical coupling is achieved (Fig. 5). On the other hand, while the gap between the waveguide and the ring is smaller than the critical distance, the ring is over-coupled. On the contrary, when the waveguide-ring gap is larger than the critical distance, the ring is under-coupled. In both last scenarios (under-coupling and over-coupling regimes) the intensity trough is shallower than that of the critical coupling point [28].

As a consequence, while the ring is positioned at a specific point, the distance variation between the ring and the waveguide changes the coupling parameter and thus the resonance transmission. Note that this gap variation not only changes the transmission coefficient, but also affects the ring effective radius (the distance between the ring center and the waveguide). Therefore, as can be seen in Fig. 6, the magnitude of the resonance trough as well as its central wavelength are changed as a result of the distance variation between the main waveguide and the 
ring. Hence, the optical sensing system converts the mechanical displacement of the ring into a shift of the resonance transmission and of its central wavelength.

According to the above explanations, the RR should be designed to have the maximum sensing range, while it is coupled to the MEMS transducer. For this purpose, the linear input measurement range of the RR should match the output mechanical range of the transducer to provide the maximum possible measurement range and an appropriate sensitivity for the proposed BioMEMS sensor. By considering these constraints, the geometrical characteristics of the RR structure are designed as summarized in Table 2.

\section{Results and Discussion}

In this section, the simulation results of the proposed biosensor are carried out by FEM methods for the MEMS transducer and FDTD methods for the optical sensing system. Based on the obtained results, the functional characteristics of the proposed optical BioMEMS sensor are then reported.

\subsection{Simulation Results of the MEMS Part}

The BioMEMS transducer behavior is studied and simulated in this sub-section, while the target molecules are exposed to the biosensor. In this scenario, surface stresses, modeled as torques, are produced on the proposed biosensor surface because of the interaction of the target molecules and the bioreceptors coated on the biosensor surface. To simulate this interaction, equivalent surface stresses (i.e. the mechanical torques) are applied on the edge of the movable beam as shown in Fig. 7a. These equivalent torques, representing the surface stress on the biosensor, cause beam deformations which result in various displacements of the center of the beam along Z-axis. In this simulation, the range of the applied torque is chosen to be varied from 0 to $1 \mathrm{~N} / \mathrm{m}$.

One of the important parameters of the BioMEMS transducer is its mechanical sensitivity which can be defined as the ratio of the displacement of the beam center to the surface stress. This parameter depends mainly on the stiffness of the movable beam which is relied on the beam geometrical dimensions. The displacement of the center of the beam and the mechanical sensitivity of the proposed biosensor as the functions of the applied forces are illustrated in Figs. 8 and 9 for various values of the beam length and its width, respectively. As can be seen in these figures, the longer and narrower the beam is, the more sensitive the proposed biosensor is. Based on the geometrical characteristics of the transducer presented in Table 1, mechanical sensitivity of $S_{m}=353 \frac{\mathrm{nm}}{(\mathrm{N} / \mathrm{m})}$ can be achieved for the present BioMEMS sensor.

Another important point related to the BioMEMS transducer is the frequency response which determines the operating bandwidth of the device. For analyzing the frequency response, one can calculate the lower order modes of the transducer 


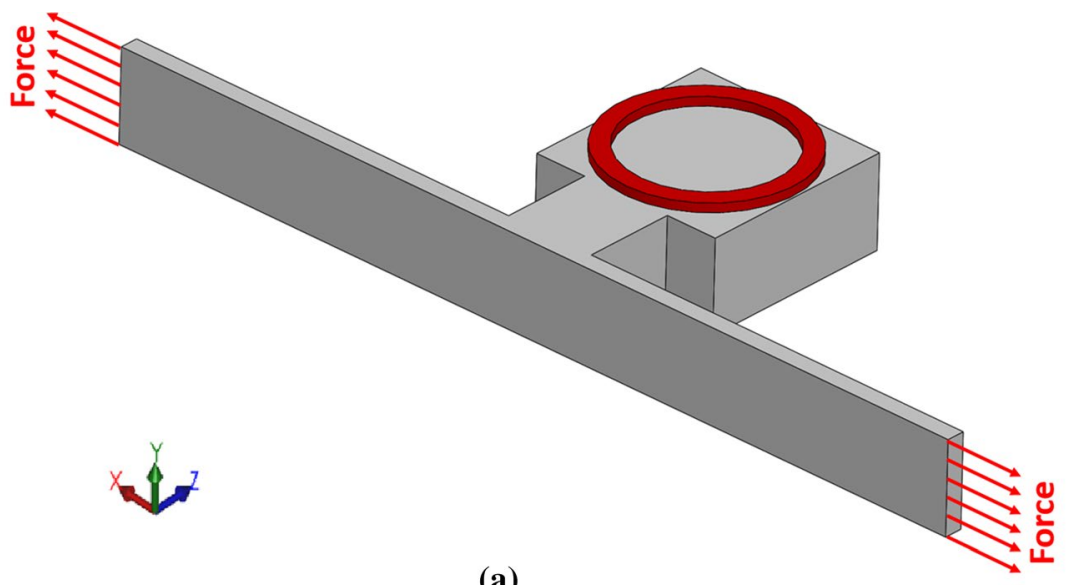

(a)

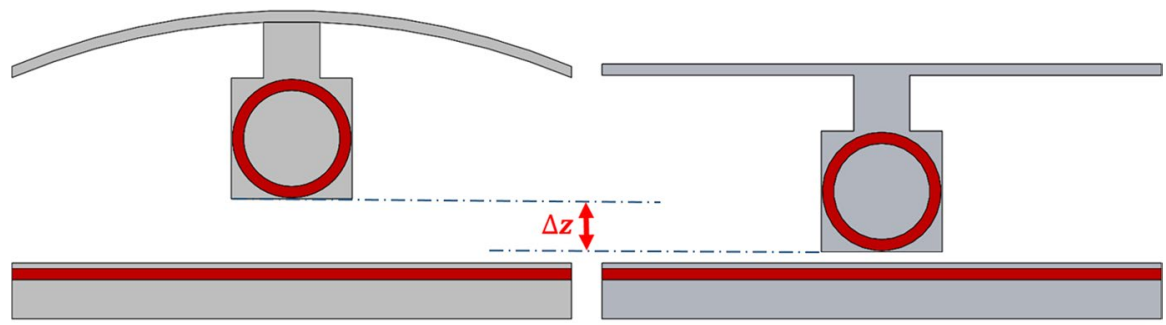

(b)

Fig. 7 a Schematics of the effects of the target molecules while they interact with the movable beam. This interaction is simulated by applying the equivalent edge forces, $\mathbf{b}$ Deformation of the movable beam under surface stresses, assuming that the beam experiences compressive surface stress, while the target molecules are absorbed by the biosensor surface

by using modal analysis. Figure 10 shows the six first mechanical mode shapes of the proposed biosensor. As shown in this figure, the first resonance mode of the mechanical transducer corresponds to a linear displacement of the center of the beam along the sensing axis $\left(f_{r}=97.78 \mathrm{kHz}\right)$. The other higher order modes cause the non-linear displacements of the beam which are not desired in this proposed biosensor. It means that, for frequencies below the first mode, the displacement of the ring provides only the z-component and zero for the other components. The Frequency response of the proposed sensor for the frequencies near the first resonance mode is shown in Fig. 11. As can be seen in this figure, the frequency response curve is relatively flat over a frequency range of 0 to $5 \mathrm{kHz}$. This range can be thus considered as the operating bandwidth of the proposed sensor (B.W $=5 \mathrm{kHz})$. 


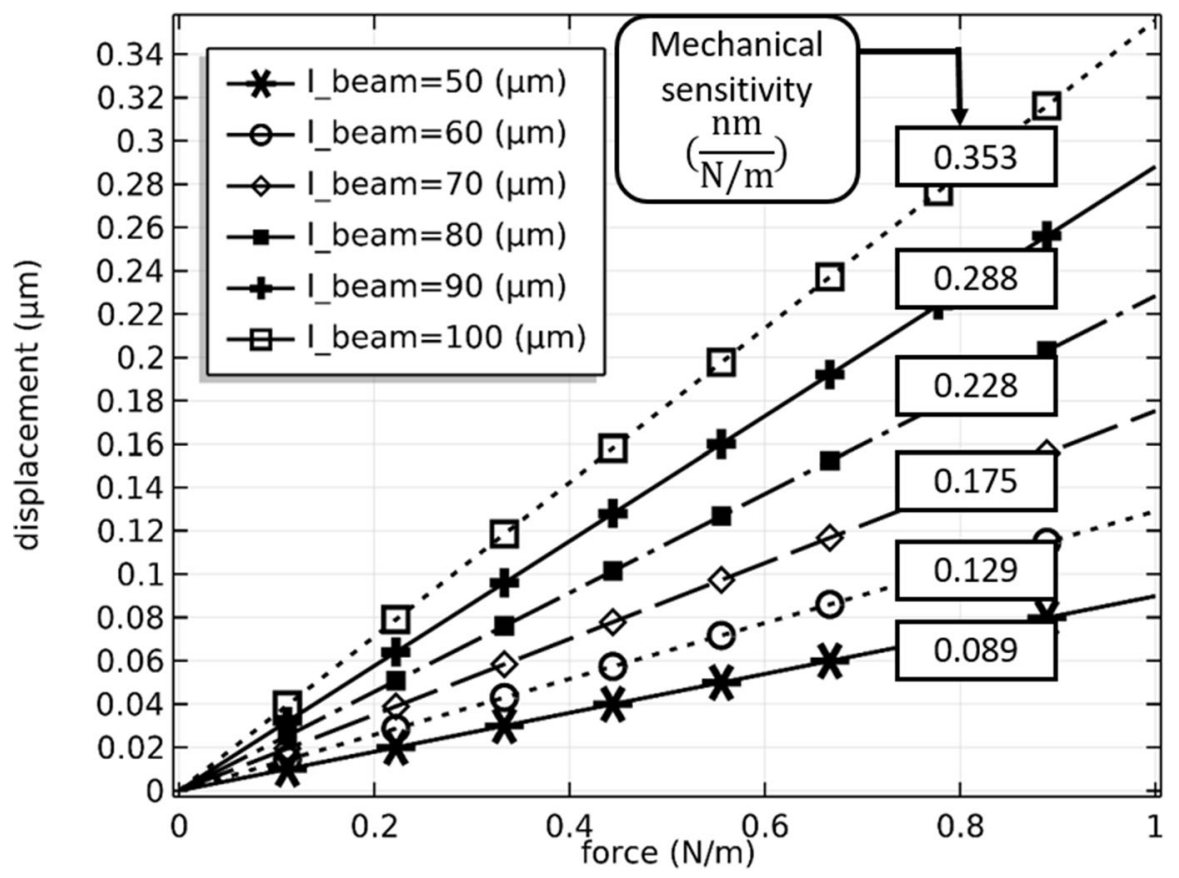

Fig. 8 Displacement of the movable beam according to the exerted force for various lengths of the beam

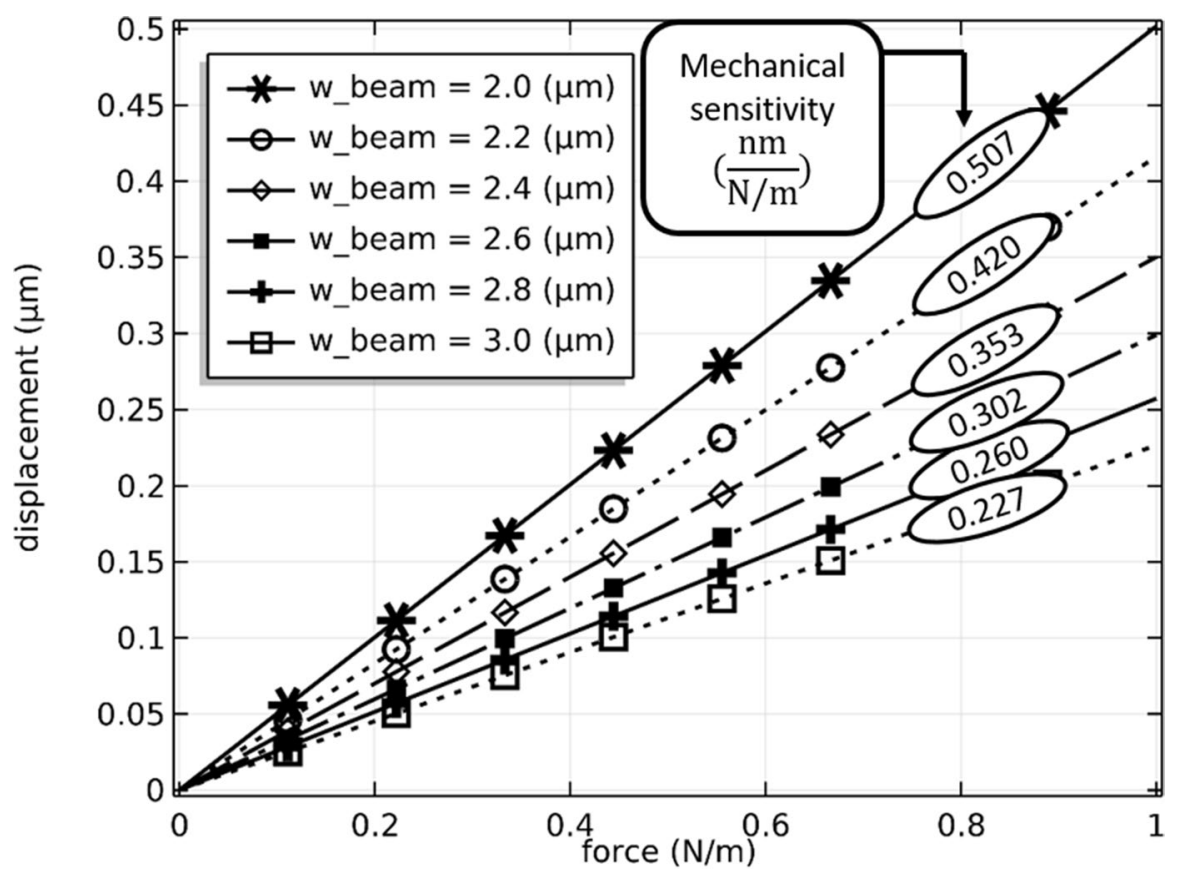

Fig. 9 Displacement of the movable beam according to the exerted force for different widths of the beam 

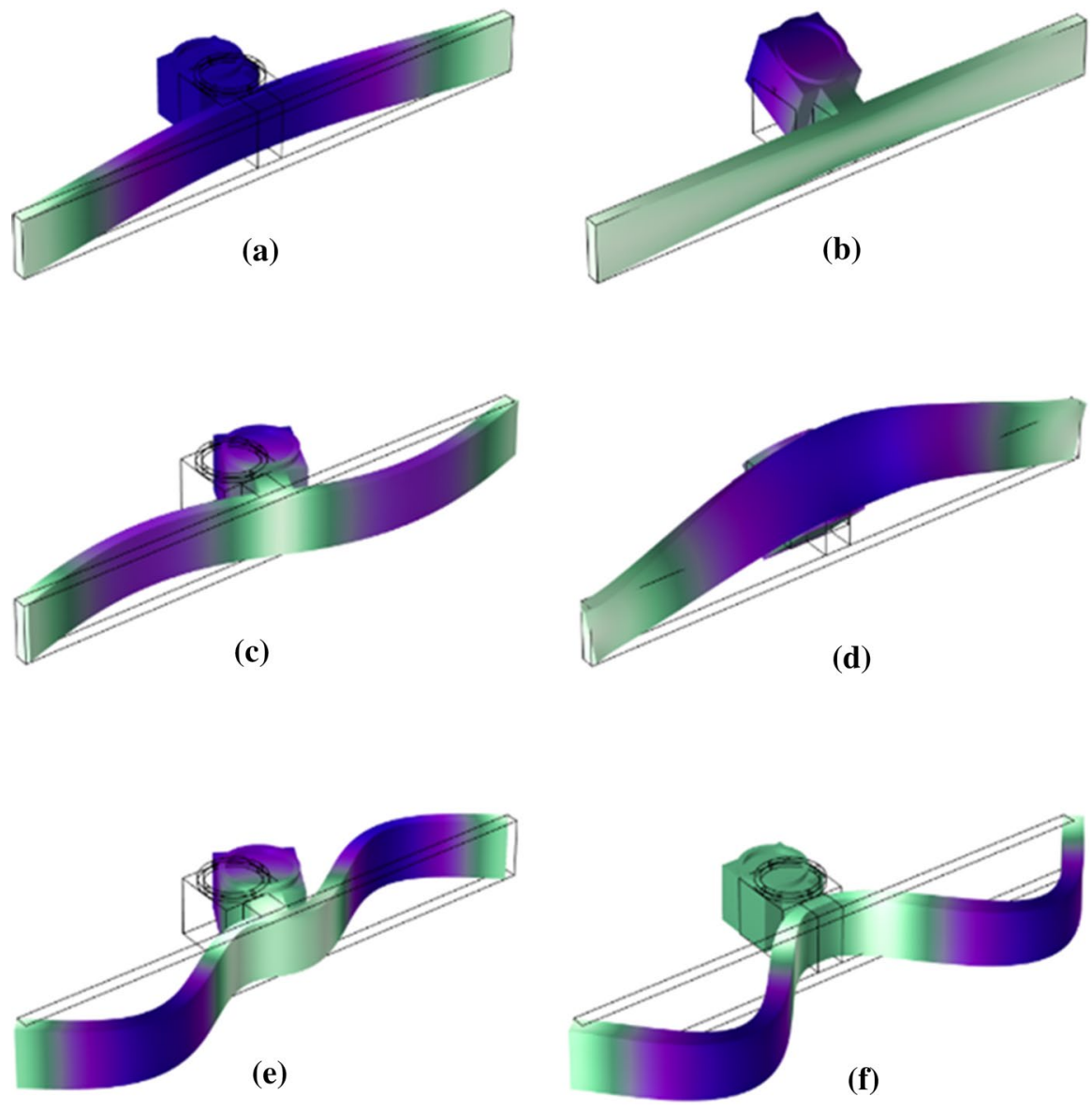

Fig. 10 First six resonance modes. a 1st mode: $f_{1}=97.78 \mathrm{kHz}, \mathbf{b}$ 2nd mode: $f_{2}=205.94 \mathrm{kHz}$, c 3rd mode: $\mathrm{f}_{3}=266.84 \mathrm{kHz}$, d 4th mode: $\mathrm{f}_{4}=895.25 \mathrm{kHz}$, e 5 th mode: $\mathrm{f}_{5}=921.39 \mathrm{kHz}, \mathbf{f}$ 6th mode: $\mathrm{f}_{6}=1106.1 \mathrm{kHz}$

\subsection{Optical Simulation Results}

As mentioned before, an optical RR is used in the proposed biosensor to measure the displacement of the ring. The initial distance between the ring and the main waveguide has been designed as $\mathrm{d}=350 \mathrm{~nm}$ to provide the maximum coupling between the main waveguide and the ring (i.e. critical coupling). Thus, there is a periodic pattern of resonant wavelengths that are filtered from the transmittance spectrum of the main waveguide, as shown in Fig. 12. As can be seen in this figure, one of these resonant troughs is measured as the sensing mode of the proposed biosensor. Furthermore, the transmission spectrums of the RR for different values of the coupling distance are illustrated 


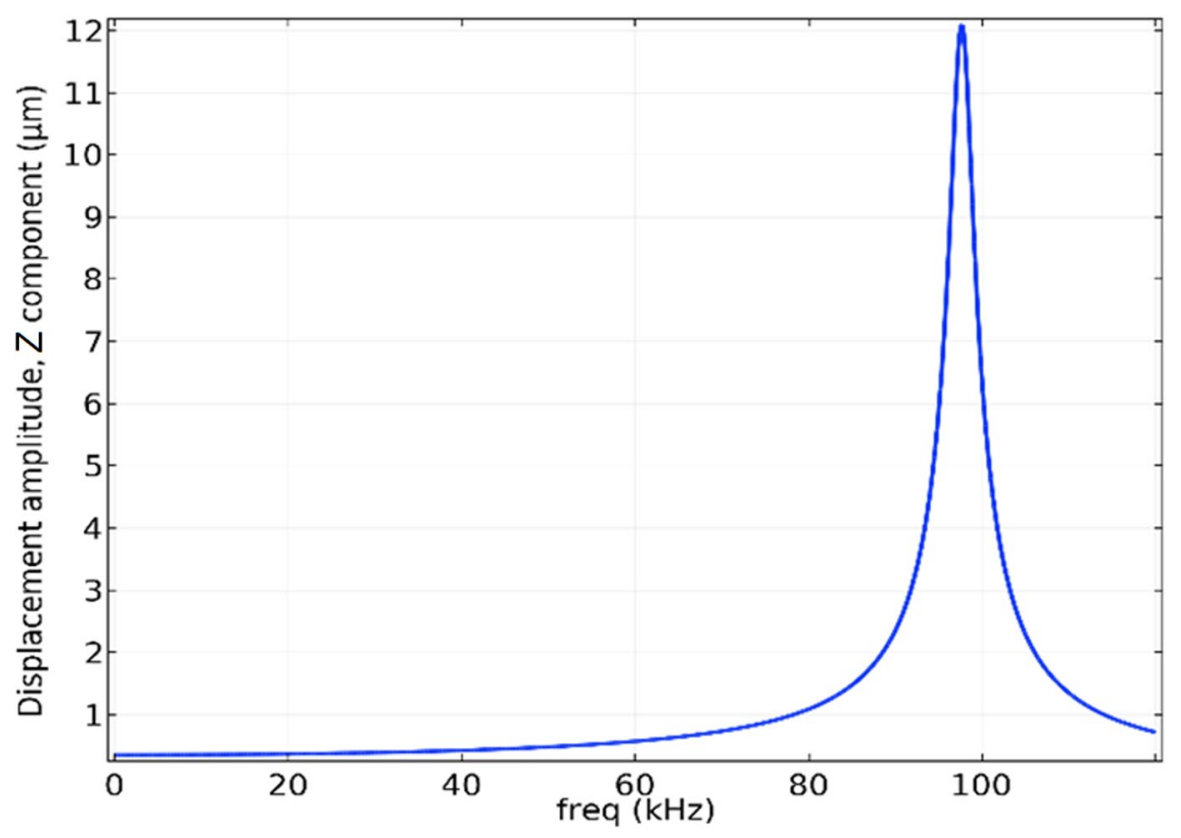

Fig. 11 Frequency response of the proposed BioMEMS sensor

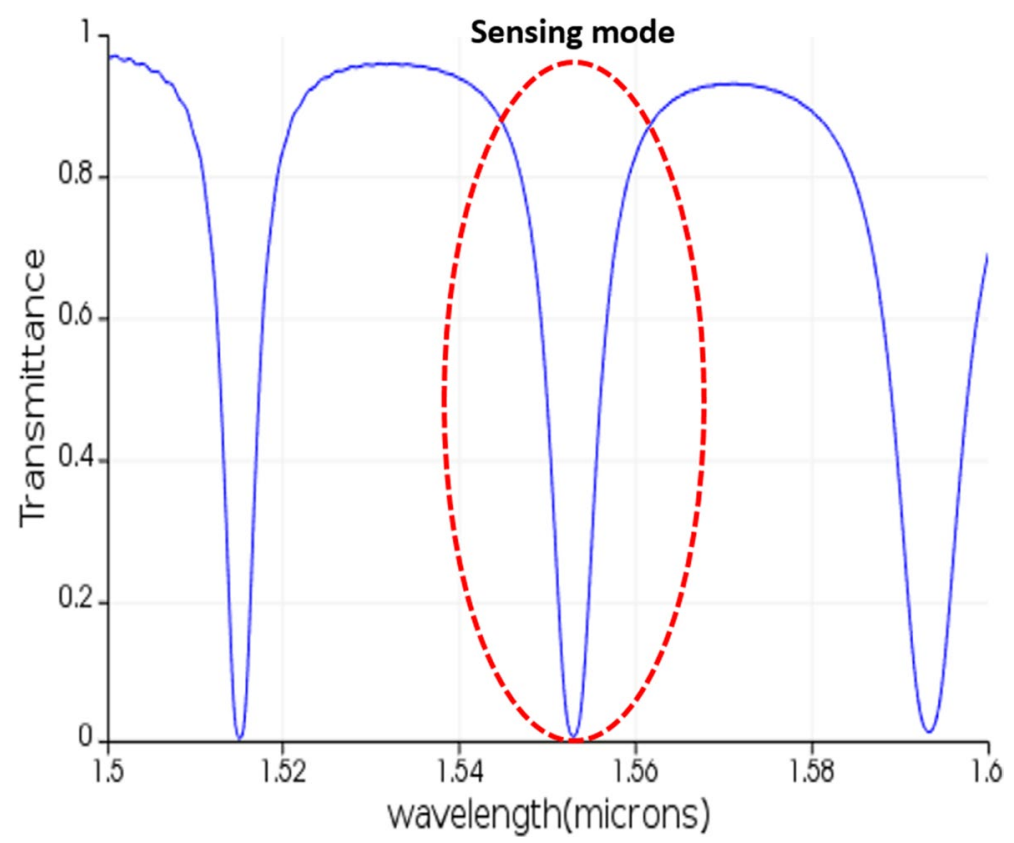

Fig. 12 The transmittance spectrum of the proposed sensor vs. wavelength. Note that critical coupling is obtained, by considering the distance between the main waveguide and the ring as $\mathrm{d}=350 \mathrm{~nm}$ 


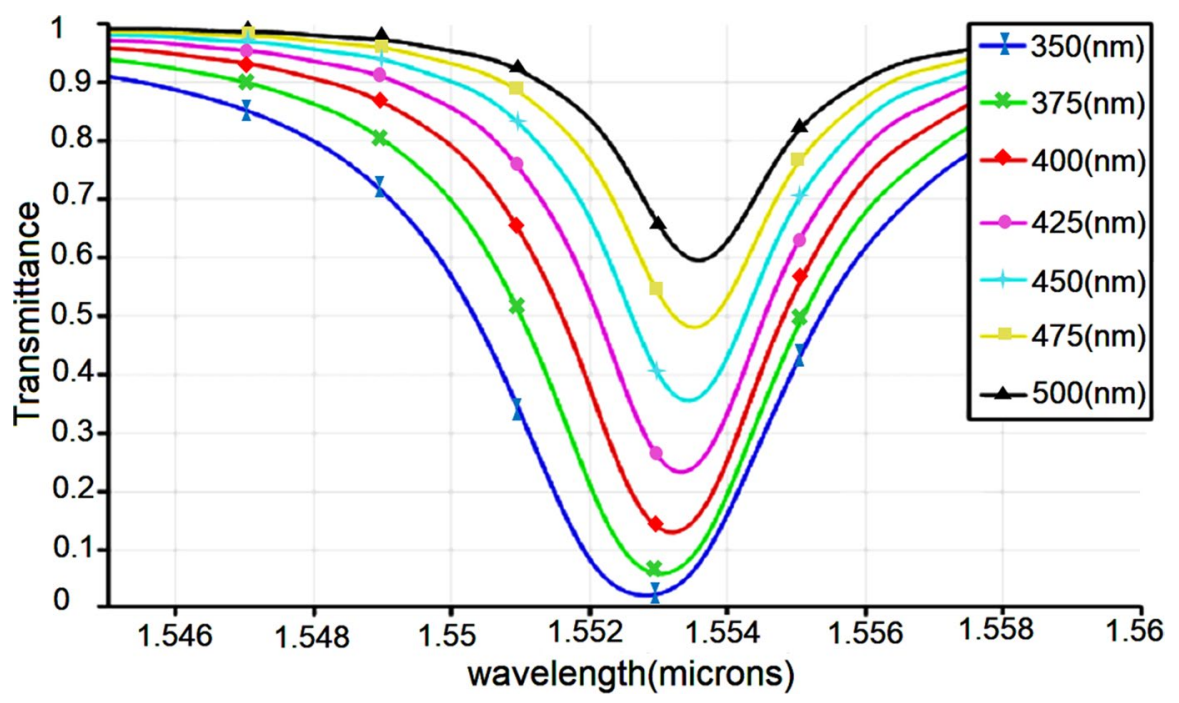

Fig. 13 Evaluation of the output transmittance spectrum for the different values of the coupling distance

in Fig. 13. As shown in this figure, the depth of the resonance trough decreases, while the gap gets wider. According to the results obtained in the two last figures, the type and the numbers of the target molecules (adsorbed by the biosensor) are determined by measuring the changes in depth and the central wavelength of the resonance trough, because these changes are a function of the gap size $(d)$. Consequently, one can diagnose the target disease and its progress level based on the type and the number of the target molecules. Finally, the optical sensitivity of this biosensor can be also defined as the ratio of the resonance trough shift to the variations of gap size. This parameter is calculated as $S_{o}=0.004 \mathrm{~nm}^{-1}$. Note that the proposed biosensor can be appropriately manufactured by nanofabrication processes including Ebeam lithography and RIE (reactive ion etching) steps.

\section{Conclusions}

In this paper, a novel optical BioMEMS sensor has been presented for the diagnosis of very small biomolecules such as cells, ions, DNAs, proteins. An optical RR structure connected to a MEMS transducer is proposed in this biosensor in order to detect the types and the numbers of the target molecules in a test sample which are corresponding to the target disease and its progress level. The simulations and the analysis of the present biosensor have been carried out by FEM approaches for the MEMS part and by FDTD methods for the optical part. Therefore, the mechanical and the optical sensitivity of the designed biosensor are respectively obtained as $S_{m}=353 \frac{\mathrm{nm}}{(\mathrm{N} / \mathrm{m})}$ and $S_{o}=0.004 \mathrm{~nm}^{-1}$. Furthermore, the frequency response of the biosensor is studied by using modal analysis to obtain its operating bandwidth as $5 \mathrm{kHz}$. According to the obtained functional characteristics of the proposed 
BioMEMS sensor, it can be appealing for highly sensitive diagnostics applications. Furthermore, it is possible to multiplex several numbers of the proposed biosensor which are coated with various bioreceptors on their surface in order to simultaneously detect various types of diseases from a sample test.

Authors' contributions All authors had equal contribution to the study conception and design.

Funding None.

\section{Declarations}

Conflicts of interest The authors declare that they have no conflict of interest.

\section{References}

1. Sheikhaleh, A., Jafari, K., \& Abedi, K. (2018). Design and analysis of a novel MOEMS gyroscope using an electrostatic comb-drive actuator and an optical sensing system. IEEE Sensors Journal, 19(1), 144-150.

2. Legendre, O., Bertin, H., Mathias, H., Megherbi, S., Juillard, J., Colinet, E., \& Jafari, K. (2013). A low-cost built-in self-test method for thermally actuated resistive MEMS sensors. Sensors and Actuators A: Physical, 194, 8-15.

3. Sheikhaleh, A., Abedi, K., Jafari, K., \& Gholamzadeh, R. (2016). Micro-optoelectromechanical systems accelerometer based on intensity modulation using a one dimensional photonic crystal. Applied Optics, 55(32), 8993-8999.

4. Jafari, K. (2017). A parameter estimation approach based on binary measurements using maximum likelihood analysis-application to MEMS. International Journal of Control, Automation and Systems, 15(2), 716-721.

5. Lee, T.M.-H. (2008). Over-the-counter biosensors: Past, present, and future. Sensors, 8(9), 5535-5559.

6. Marvi, F., \& Jafari, K. (2021). A measurement platform for label-free detection of biomolecules based on a novel optical BioMEMS sensor. IEEE Transactions on Instrumentation and Measurement, 70, 1-7.

7. Coffel, J., \& Nuxoll, E. (2018). BioMEMS for biosensors and closed-loop drug delivery. International Journal of Pharmaceutics, 544(2), 335-349.

8. Yoon, J., Shin, M., Lee, T., \& Choi, J. W. (2020). Highly sensitive biosensors based on biomolecules and functional nanomaterials depending on the types of nanomaterials: A perspective review. Materials, 13(2), 299.

9. Castillo-Henríquez, L., Brenes-Acuña, M., Castro-Rojas, A., Cordero-Salmerón, R., LoprettiCorrea, M., \& Vega-Baudrit, J. R. (2020). Biosensors for the detection of bacterial and viral clinical pathogens. Sensors, 20(23), 6926.

10. Choi, J. R. (2020). Development of point-of-care biosensors for COVID-19. Frontiers in Chemistry, 8, 517.

11. Gopinath, P., Anitha, V., \& Mastani, S. A. (2015. Microcantilever based biosensor for disease detection applications. Journal of Medical and Bioengineering, 4(4), 34.

12. Saeed, M. A., Khan, S. M., Ahmed, N., Khan, M. U., \& Rehman, A. (2016). Design and analysis of capacitance based Bio-MEMS cantilever sensor for tuberculosis detection. In 2016 International conference on intelligent systems engineering (ICISE), 2016 (pp. 175-180).

13. Ansari, M. Z., Bisen, M., \& Cho, C. (2018). Modelling and analysis of diaphragm integrated SU8/CB nanocomposite piezoresistive polymer microcantilever biosensor. Microsystem Technologies, 24(7), 3061-3068. 
14. Hernández-Sebastián, N., Díaz-Alonso, D., Renero-Carrillo, F. J., Villa-Villaseñor, N., \& Calleja-Arriaga, W. (2018). Design and simulation of an integrated wireless capacitive sensors array for measuring ventricular pressure. Sensors, 18(9), 2781.

15. Filippidou, M., Chatzichristidi, M., \& Chatzandroulis, S. (2019). A fabrication process of flexible IDE capacitive chemical sensors using a two step lift-off method based on PVA patterning. Sensors and Actuators B: Chemical, 284, 7-12.

16. Zhang, L., Rahimabady, M., Tan, S. Y., Tan, C. Y., Chen, S., Chen, Y. F., et al. (2017). P (VDFHFP) polymer as sensing material for capacitive carbon dioxide sensors. IEEE Sensors Journal, 17(14), 4349-4356.

17. Kim, D.-S., Jeong, Y.-J., Lee, B.-K., Shanmugasundaram, A., \& Lee, D.-W. (2017). Piezoresistive sensor-integrated PDMS cantilever: A new class of device for measuring the drug-induced changes in the mechanical activity of cardiomyocytes. Sensors and Actuators B: Chemical, 240, 566-572.

18. Takahashi, T., Hizawa, T., Misawa, N., Taki, M., Sawada, K., \& Takahashi, K. (2018). Surface stress sensor based on MEMS Fabry-Perot interferometer with high wavelength selectivity for label-free biosensing. Journal of Micromechanics Microengineering, 28(5), 054002.

19. Li, C., Zhang, G., Wu, S., \& Zhang, Q. (2018). Aptamer-based microcantilever-array biosensor for profenofos detection. Analytica Chimica Acta, 1020, 116-122.

20. Pohanka, M. (2018). Overview of piezoelectric biosensors, immunosensors and DNA sensors and their applications. Materials, 11(3), 448.

21. Takahashi, K., Fujie, T., Teramoto, R., Takahashi, I., Sato, N., Takeoka, S., et al. (2019). Elastomerbased MEMS optical interferometric transducers for highly sensitive surface stress sensing for biomolecular detection. MRS Communications, 9(1), 381-389.

22. Serene, I. M., \& Alex, Z. C. (2016). Optical MEMS sensor for measurement of low stress using Ptolemy II. Advances in Systems Science Applications, 16(3), 76-93.

23. Zhu, S., Li, H., Yang, M., \& Pang, S. W. (2016). High sensitivity plasmonic biosensor based on nanoimprinted quasi 3D nanosquares for cell detection. Nanotechnology, 27(29), 295101.

24. Cattoni, A., Ghenuche, P., Haghiri-Gosnet, A.-M., Decanini, D., Chen, J., Pelouard, J.-L., et al. (2011). $\lambda 3 / 1000$ plasmonic nanocavities for biosensing fabricated by soft UV nanoimprint lithography. Nano Letters, 11(9), 3557-3563.

25. Sreekanth, K. V., Alapan, Y., ElKabbash, M., Ilker, E., Hinczewski, M., Gurkan, U. A., et al. (2016). Extreme sensitivity biosensing platform based on hyperbolic metamaterials. Nature Materials, 15(6), 621-627.

26. Grafov, B. (1999). The Shuttleworth equation for a finite surface strain. Journal of Electroanalytical Chemistry, 471(2), 105-108.

27. Sang, S., Zhao, Y., Zhang, W., Li, P., Hu, J., \& Li, G. (2014). Surface stress-based biosensors. Biosensors and Bioelectronics, 51, 124-135.

28. Demirtzioglou, I., Lacava, C., Bottrill, K. R., Thomson, D. J., Reed, G. T., Richardson, D. J., et al. (2018). Frequency comb generation in a silicon ring resonator modulator. Optics express, 26(2), $790-796$.

Publisher's Note Springer Nature remains neutral with regard to jurisdictional claims in published maps and institutional affiliations. 